\title{
The Nucleation and Wetting Behavior at Hydrophilic, Polyacrylate Nanostructures Fabricated via Direct Laser Writing
}

\author{
Andreas Verch $^{1} *$, Julia Purtov ${ }^{1,2}$ and Niels de Jonge ${ }^{1,3}$ \\ 1. INM - Leibniz Institute for New Materials, Campus D2 2, 66123 Saarbrücken, Germany. \\ 2. Department of Materials Science and Engineering, Saarland University, Campus D2 2, 66123 \\ Saarbrücken, Germany. \\ 3. Department of Physics, University of Saarland, Campus A5 1, 66123 Saarbrücken, Germany. \\ *Corresponding author e-mail address: andreas.verch@leibniz-inm.de
}

In the area of controlled liquid movement, surfaces providing a pronounced wetting behavior are of particularly interest [1]. A look for inspiration into nature reveals a broad variety of water repellent and water attracting surfaces [2]. Prominent representatives are the super-hydrophobic eyes of moths [3] and the water pinning petals of roses [4]. While the former were intensively investigated over the last decades due to an obvious application in anti-fogging coatings, the interest in the latter rose only recently mainly driven by its significance for microfluidics and structural genomics [5-7]. Here, the pinning behavior of surfaces was macroscopically characterized by using static or dynamic contact angle experiments. However, no detailed nucleation investigations on micro-scale have been performed so far. In this work we want to focus exactly on this missing component.

Toward this aim, we used a two photon lithography system to fabricate pillar structures in different dimensions. The pillars were arranged in a square pattern. The distances separating individual pillars were $0.5,1,2,5$ and $10 \mu \mathrm{m}$. The structures, which consist of a hydrophilic, acrylic co-polymer (Figure 1A), were written on glass surfaces covered with an adhesion promoter showing a water contact angle of $80^{\circ}$. Interestingly, the effective contact angle of water in the pillar area was larger than $110^{\circ}$ indicating hydrophobic wetting behavior, although the glass substrate and the polymer were both hydrophilic (Figure 1B).

Condensation and wetting experiments were conducted using an environmental scanning electron microscope (ESEM). The on glass slides fabricated structures were cooled to $1^{\circ} \mathrm{C}$. At this temperature, water already condensates at a water vapor pressure of $657 \mathrm{~Pa}$. By varying vapor pressure we could determine the supersaturations required to precipitate water on unstructured and differently structured surfaces. In addition, the nucleation density and its dependency on the dimensions of the structures could be determined. Exemplarily the effect of the surface structuring on the nucleation density is illustrated in Figure 1C). On the smooth, unstructured slightly hydrophilic glass surface, larger but only few droplets of water precipitated. In contrast to that, significantly more individual water droplets appeared in the structured area. These droplets, though, are considerably smaller and appeared to be pinned to and predominantly delimited by the arrangement of the pillars (Figure 2A). The pinning effect is also observed on the macroscopic scale (Figure 2B). Usually, surfaces featuring structures separated by a distance in the range of visible light have diffracting properties. In the case of our pillars this is displayed by a red coloration of the structured area. If liquid is applied to the surface, though, the coloration turned to transparent indicating a filling of the areas between the pillars by the liquid. During the evaporation a thin film of liquid remained pinned by the structures while the volume of the droplet decreased continuously. 
In this work we show that structuring of surfaces has a huge influence on the wetting and nucleation behavior of surfaces [8].

\section{References:}

[1] L Feng et al, Advanced Materials, 14 (2002), p. 1857.

[2] R Hensel, C Neinhuis and C Werner, Chemical Society Reviews, 45 (2016), p. 323.

[3] H Peisker and SN Gorb, Journal of Experimental Biology 213 (2010), p. 3457.

[4] L Feng et al, Langmuir 24 (2008), p. 4114.

[5] JBK Law et al, Langmuir 30 (2014), p. 325.

[6] J Xi and L Jiang, Industrial \& Engineering Chemistry Research 47 (2008), p. 6354.

[7] MK Dawood et al, Langmuir, 27 (2011), p. 4126.

[8] We thank E. Arzt for his support through INM.

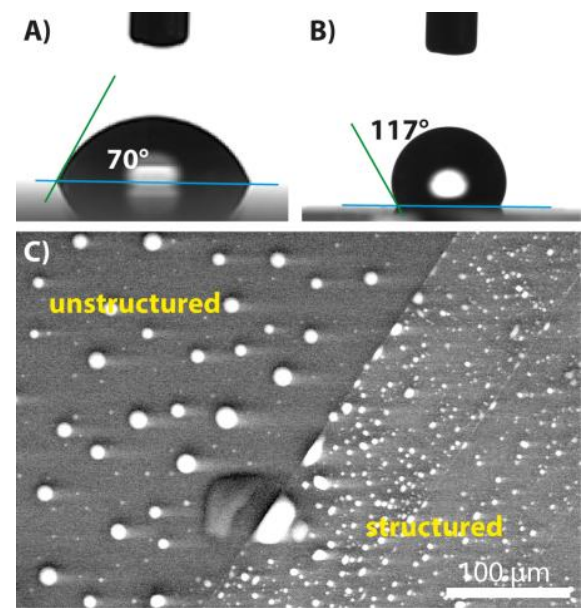

Figure 1. Condensation and wetting behavior of the unstructured and the pillar structured hydrophilic surface. A) and B) show the contact angle measurements of the unstructured, smooth polymer and the surface structured with pillars, respectively. The presence of pillars increases the contact angle to $117^{\circ}$. C) Differences in the wetting between the unstructured (smooth) glass surface and the structured surface (pillars).
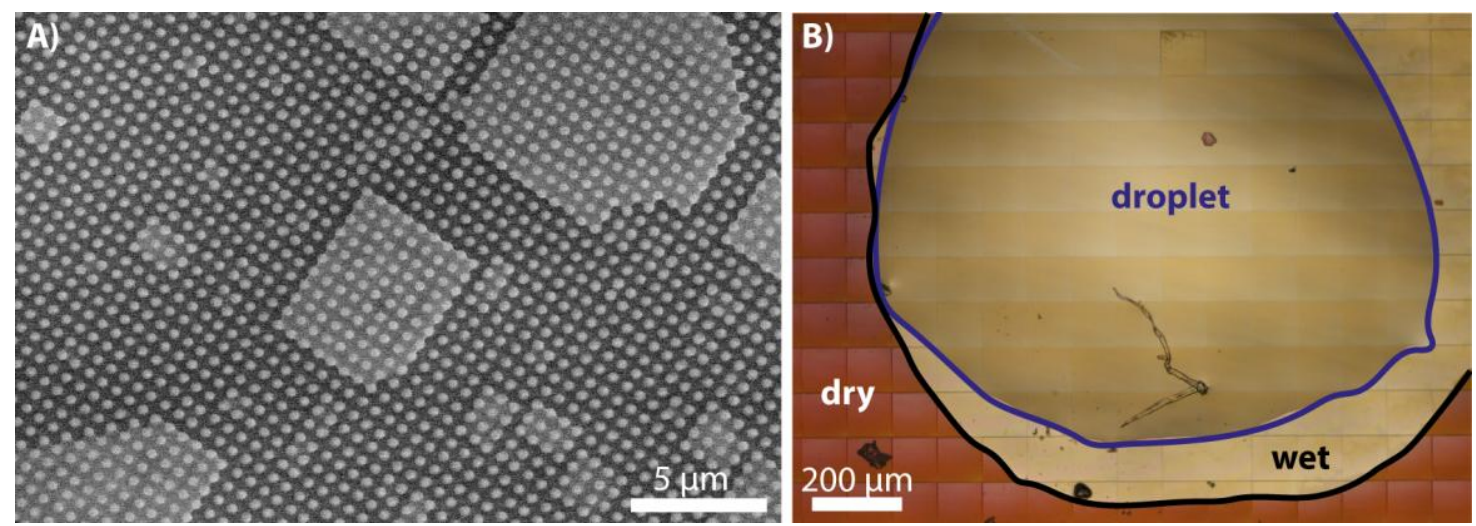

Figure 2. Pinning of liquid to the pillar structures. A) ESEM image illustrating the correlation of the droplet shape to the pillar arrangement. B) Optical microscopy image displaying macroscopic droplet pinning. During the evaporation, a thin film of liquid remains pinned by the structures (black encircled), while the volume of the droplet decreases continuously (blue encircled). 